\title{
Pulmonary endarterectomy: technique and pitfalls
}

\author{
Stefan Guth, Eckhard Mayer, Diethard Prüfer, Christoph B. Wiedenroth \\ Department of Thoracic Surgery, Kerckhoff Heart and Thorax Center, Bad Nauheim, Germany \\ Correspondence to: Priv.-Doz. Dr. med. Stefan Guth, MD. Department of Thoracic Surgery, Kerckhoff Heart and Thorax Center, Benekestr. 2-8, \\ 61231 Bad Nauheim, Germany. Email: s.guth@kerckhoff-klinik.de.
}

\begin{abstract}
Chronic thromboembolic pulmonary hypertension (CTEPH) remains a rare and underdiagnosed disease. After one or several episodes of acute pulmonary embolism, around $3 \%$ of patients develop CTEPH and two-thirds of these patients are potential surgical candidates. Besides surgery, additional treatment modalities are pulmonary arterial hypertension medication and balloon pulmonary angioplasty. Patients should be evaluated in CTEPH expert centers to ensure the most appropriate therapy. Pulmonary endarterectomy (PEA) is a complex, but standardized surgical procedure aiming to clear the obstructed pulmonary arteries completely. For optimal visualization, deep hypothermic circulatory arrest is a prerequisite. This article will give an overview of the evaluation, indication and surgical management of patients with CTEPH.
\end{abstract}

Keywords: Chronic thromboembolic pulmonary hypertension (CTEPH); pulmonary endarterectomy (PEA); balloon pulmonary angioplasty (BPA)

Submitted Oct 15, 2021. Accepted for publication Jan 17, 2022.

doi: 10.21037/acs-2021-pte-185

View this article at: https://dx.doi.org/10.21037/acs-2021-pte-185

\section{Introduction}

Chronic thromboembolic pulmonary hypertension $(\mathrm{CTEPH})$ is a rare and underdiagnosed disease that can often be treated curatively by pulmonary endarterectomy (PEA) $(1,2)$. The contemporary treatment of CTEPH patients is based on three modalities: Pulmonary endarterectomy as a complex surgical procedure, pulmonal arterial hypertension (PAH) pharmacotherapy and balloon pulmonary angioplasty (BPA) as a demanding interventional procedure. For patients with technically accessible pulmonary arterial obstructions, PEA with surgical removal of the fibrous tissue remains the gold standard treatment with excellent short and long-term results (3). If patients are not deemed amenable for surgery, PAH pharmacotherapy +/- BPA are considered treatment possibilities. For optimal treatment decision-making, patients should be evaluated in experienced CTEPH centers by multidisciplinary teams with experienced PEA surgeons. This article highlights the surgical treatment of CTEPH by PEA.

\section{Diagnostic work-up}

The prerequisite for sound decision-making regarding a proper therapeutic concept in CTEPH patients is a thorough clinical and hemodynamic evaluation in addition to high-quality imaging of the pulmonary vasculature. For the diagnosis of $\mathrm{PH}$ and assessment of disease severity, right heart catheterization is mandatory to measure pulmonary artery pressures, pulmonary artery occlusion pressure and cardiac output. Further evaluation comprises echocardiography, body plethysmography, cardiopulmonary exercise test, coronary angiography, and carotid ultrasound.

\section{Imaging}

The imaging comprises digitally subtracted pulmonary angiography, dual source computed tomography (CT) and magnetic resonance tomography. Angio CT and pulmonary angiography deliver additional information: CT visualizes lung parenchyma and lobar to segmental vessels, while 


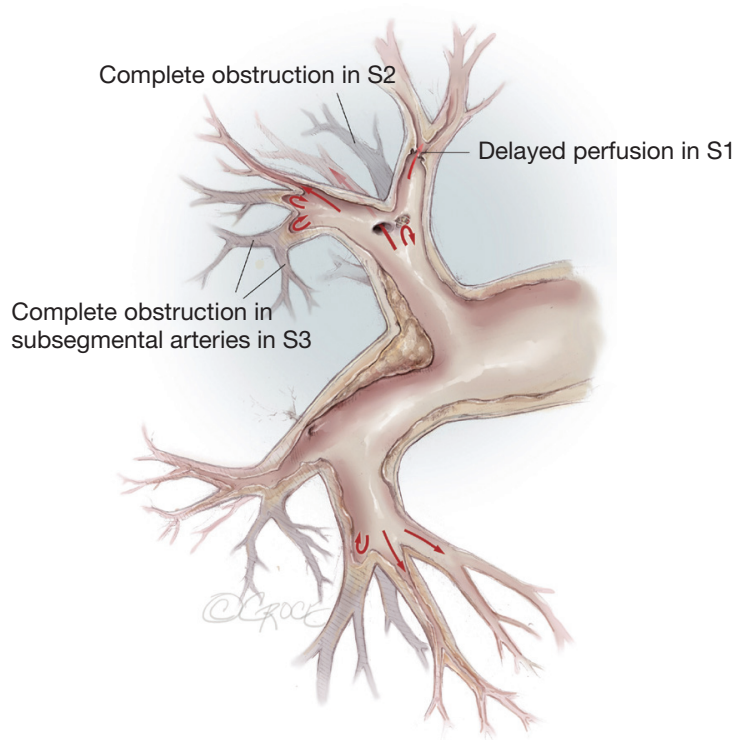

Figure 1 Schematic view of the right pulmonary artery in $30^{\circ}$ left anterior oblique view showing high grade stenoses and complete obstructions of the pulmonary artery tree.

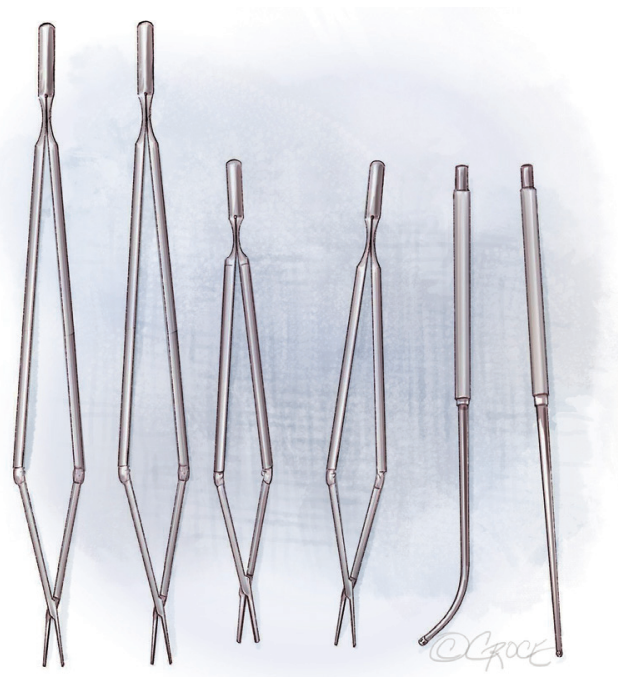

Figure 2 Dual-action forceps and suction and dissection devices (angled and straight) are important instruments for pulmonary endarterectomy.

selective angiography has advantages in peripheral vessels. For exact localization of pulmonary vascular obstructions, we use $30^{\circ}$ contralateral as well as $90^{\circ}$ ipsilateral projections for each side (Figure 1).

\section{Patient selection}

Indication for surgery in CTEPH patients depends on several factors: symptom severity, degree of $\mathrm{PH}$ and right heart dysfunction, level of stenoses and occlusions, correlation between $\mathrm{PH}$ and extent of obstructions, comorbidities and possible surgical difficulties, patient expectations and risk acceptance (4). One key aspect is the technical accessibility of the obstructive material. This is mainly dependent on surgical expertise, which correlates with the case load of the individual surgeon. The surgical risk depends on the severity of hemodynamic impairment compared to the amount of obstructive material, comorbidities, and age of the patients.

\section{Operative techniques}

\section{Special Instruments}

Meanwhile, there are special instruments for PEA. Figure 2 shows the double-action forceps which exist in two lengths and with $1-$ and $2.5-\mathrm{mm}$ tips. For raising the dissection plane, a micro-endarterectomy spatula is used and for the distal dissection, special PEA suction/dissectors in straight and angled shapes are used.

\section{Preparation}

After initiation of general anesthesia, radial and femoral artery pressure monitoring lines, a central venous line, a pulmonary arterial catheter (PAC), and a urinary catheter are placed. Before PAC placement, $17.5 \%$ of the full heparin dose $(5,000 \mathrm{U})$ needed for extracorporeal circulation (ECC), is administered to avoid development of a fresh thrombus at the catheter tip (5). Routinely, a transesophageal echocardiography (TEE) probe is inserted. The patient is positioned supine and surgical exposure is via median sternotomy from the superior border of the manubrium to the xiphoid (Figure 3). The pericardium is incised, and purse-string sutures are placed into the ascending aorta, right atrial appendage and right atrium followed by insertion of the arterial cannula and two venous cannulas, which are placed crosswise into the superior (SVC) and inferior vena cava to avoid the surgical field. Additionally, an aortic root vent and vent catheters are inserted into the pulmonary trunk and into the left atrium via the right superior pulmonary vein (Figure 4). For facilitation of exposure, loops are placed around the ascending aorta, pulmonary trunk and SVC. 


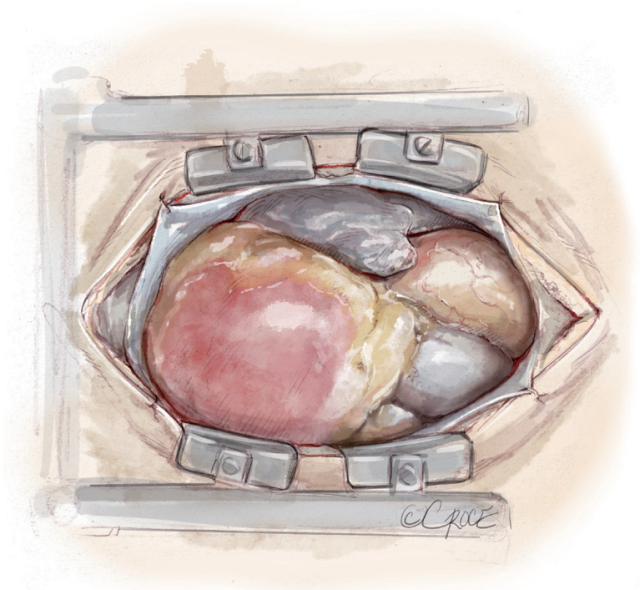

Figure 3 A sternotomy is performed from the superior border of the manubrium sterni to the xiphoid.

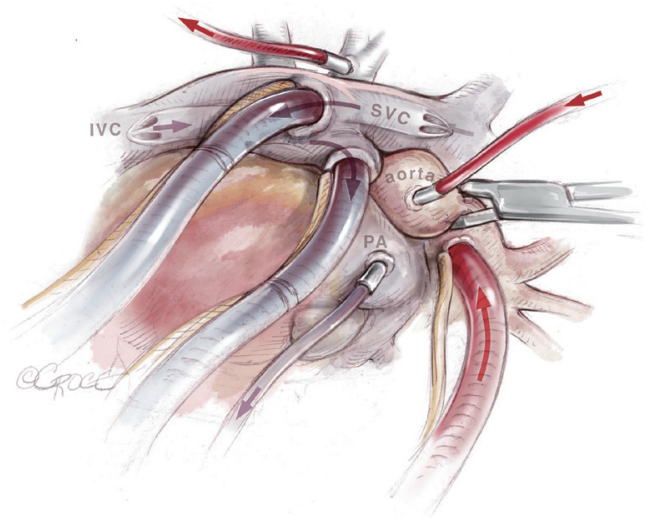

Figure 4 In preparation for the pulmonary endarterectomy, cannulas are inserted into the ascending aorta, both venae cavae (superior and inferior) from the right atrium to give adequate access to the right pulmonary artery. Additionally, vent-catheters are placed into the pulmonary trunk and into the left atrium via the right superior pulmonary vein.

\section{Exposition}

Importantly, the SVC must be mobilized by incising the pericardium down to the SVC and dissecting the vein from the right main pulmonary artery. Then a modified spreader can be placed between the ascending aorta and SVC, which is splinted by the inserted venous cannula, avoiding congestion of the upper part of the body. Below, the right main pulmonary artery is exposed. Incision with a scalpel is best performed with congestion of the pulmonary artery to

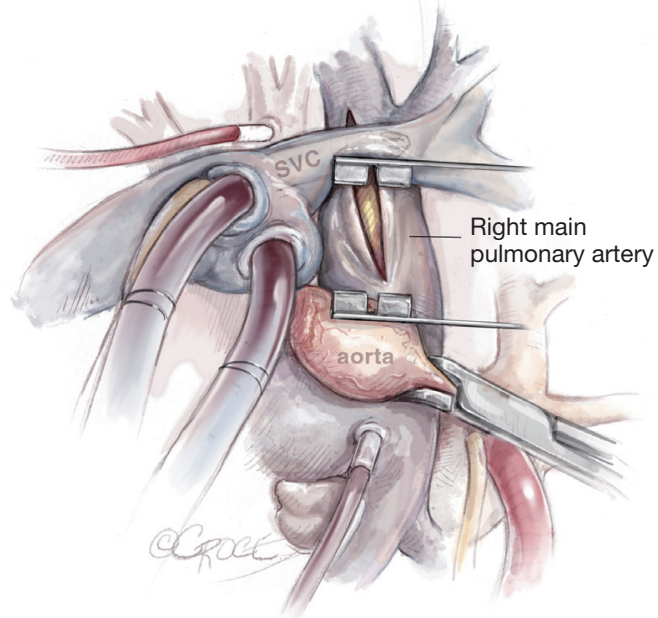

Figure 5 Access to the right pulmonary artery with a spreader between superior vena cava and ascending aorta. The superior vena cava is kept open by the inserted venous cannula.

avoid a misaligned entry. Also important is to localize the ostium of anterior trunk of the right upper lobe to avoid an incision into the trunk. Figure 5 depicts the line of incision between the aorta and SVC.

\section{Operation}

With the established ECC, the patient is cooled below $20^{\circ} \mathrm{C}$ to allow periods of deep hypothermic circulatory arrest (DHCA). For uniform cooling of the body, a time of 60 to 90 minutes is necessary. DHCA is mandatory to get a bloodless surgical field and to perform the endarterectomy down to the subsegmental branches of the pulmonary artery tree. Usually, intervals limited to 20 minutes of DHCA are sufficient for both sides to complete the endarterectomy. In technically challenging cases with predominantly distal obstructions, cumulative DHCA times up to 60 to 70 minutes, with several arrest and reperfusion periods can be necessary.

The principal concept of PEA is shown in Figure 6. From the proximal incision of the main pulmonary artery, a circumferential dissection plane must be established that is extended to the distal branches. Occasionally, gross centrally located thrombotic material must be removed first to provide adequate space to start the endarterectomy (Figure 7). Less experienced surgeons might complete the operation at this point without performing the correct 


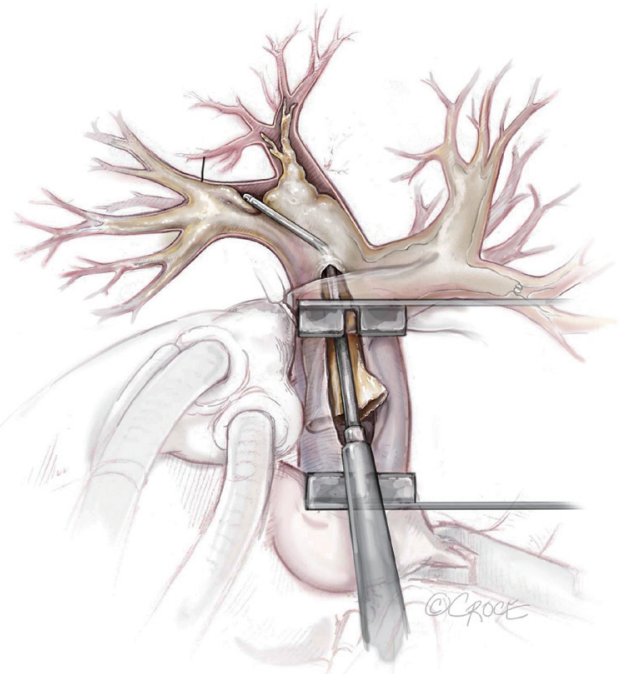

Figure 6 The principle of the endarterectomy. The incision lays in the central part of the pulmonary artery and from here the dissection plane must be prepared forward to the distal obstructing material. For distal visibility, deep hypothermic circulatory arrest is mandatory.

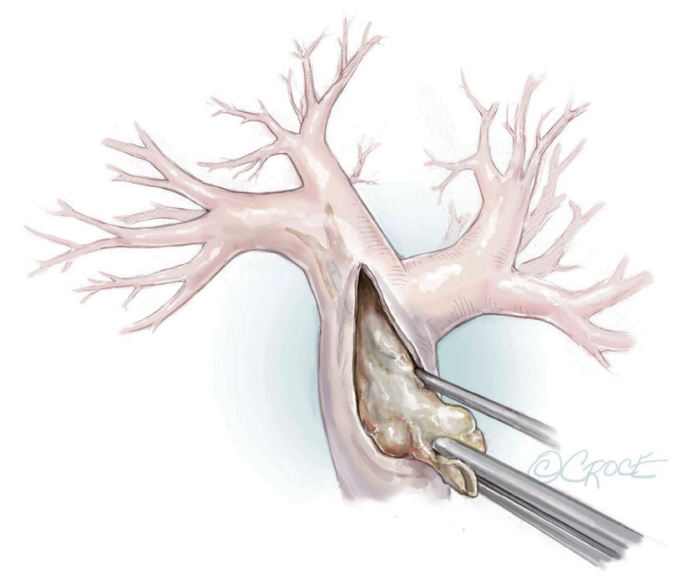

Figure 7 Removal of a centrally located bulk of obstructive material. This is only the first step and after this, the endarterectomy to the distal segmental and subsegmental artery must be performed.

endarterectomy. The key point for successful surgery is to raise the correct endarterectomy plane between the intimal and the medial layer and to perform a complete endarterectomy of all technically reachable obstructions (Figure 8). Sometimes the intimal layer is too fragile and

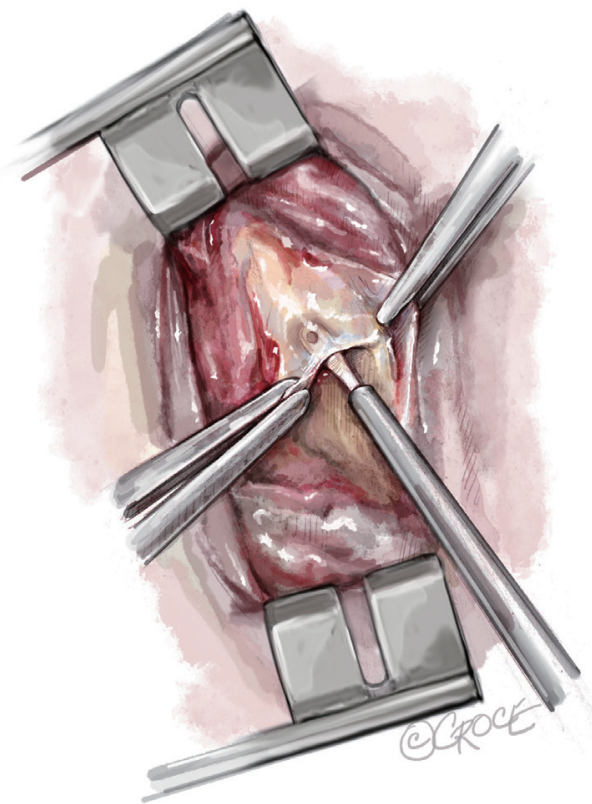

Figure 8 Raising the correct dissection plane in the proximal artery. Often it is the fragile transparent intimal layer.

the dissection plane must be extended into the medial layer to establish an adequate dissection cylinder. Here, the experience of the surgeon is pivotal to avoid a potentially fatal complete rupture of the vessel. After raising the correct plane centrally, DHCA ensues and endarterectomy is extended into all obstructed branches of the vascular tree.

The fact that technical difficulties, especially the tissue quality of the endarterectomy plane, cannot be foreseen by any imaging examination, makes PEA surgery challenging. If the endarterectomy specimen is fragile or an adequate endarterectomy plane cannot be raised in the central pulmonary artery, the surgeon must raise an endarterectomy plane where the fibrous material is just visible, even in the segmental or subsegmental arteries. Figure 9 depicts a sieve plate obstructing subsegmental arteries. In this case, the surgeon is forced to begin the dissection by grasping the fibrous material with special small double-action forceps and use the PEA suction/dissector (Figure 2) to develop a plane between the vessel wall and the obstructing material. The magnified view (Figure 9) shows the lifting of the obstructive material (i.e., sieve plate), and then wider forceps may be used to complete the dissection. These manoeuvres need experience and are challenged by the limited timeframe of DHCA, especially in difficult cases, where this must be repeated several times. 


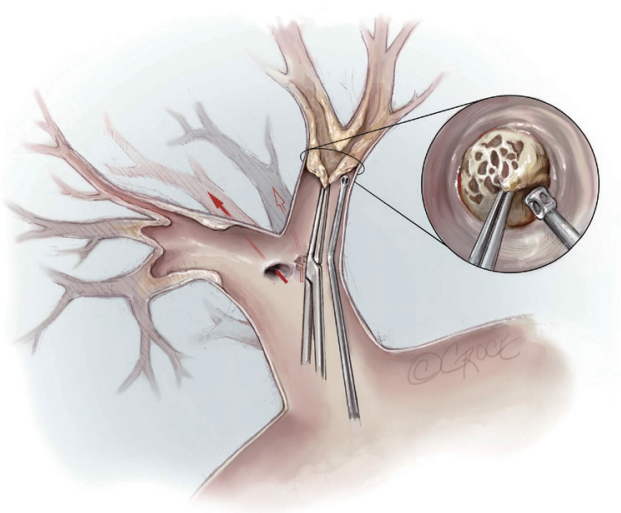

Figure 9 Regularly, the endarterectomy must be started in segmental or subsegmental arteries and makes the surgery difficult. This is illustrated here by an occlusive sieve plate in a distal artery.

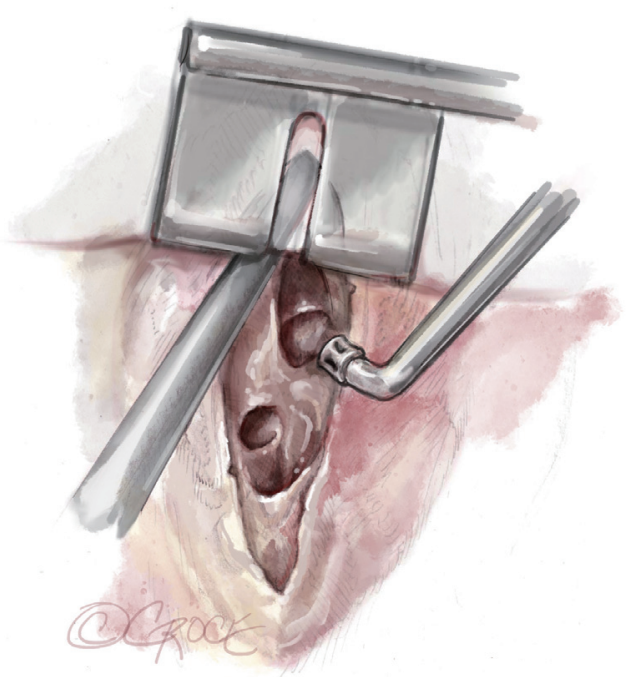

Figure 10 View inside the right artery after successful endarterectomy with clearly open ostia to the segmental arteries.

Usually, we begin with the right pulmonary artery and after the endarterectomy is completed, a bubble test is done: the lungs are inflated and the vasculature is rinsed with crystalloid solution to rule out an open connection between the alveolar space and the vasculature (6). In case of a fistula with bubbles rising from a segmental or subsegmental branch, we use fibrin glue to seal this vessel and avoid possible endobronchial hemorrhage. In stable conditions, the ECC is restored and the pulmonary artery incision is sutured by a double-armed running suture using Prolene 6-0. Then a second infusion of blood cardioplegia ensues and the same procedure is performed for the opposite pulmonary artery tree. The incision starts in the pulmonary trunk and is extended into the main left pulmonary artery. For better visibility, the loop around the pulmonary trunk is stretched caudally. Figure 10 shows the final view after successful endarterectomy with patent ostia of lobar and segmental arteries. Figure 11 depicts a typical specimen with centrally located intima and distal fibrous material at segmental or subsegmental level.

\section{Completion}

After the left side ECC is restored, the patient is re-warmed to normal body temperature. If necessary, additional cardiac procedures (closure of a patent foramen ovale, aortocoronary bypass surgery, or valve repair/replacement) can be performed during re-warming. The patient is placed in Trendelenburg position, the arterial vent catheter is started and the aortic cross-clamp removed. All loops and the left atrial vent catheter are removed and wires for the external pacemaker are placed. After complete re-warming, gradual weaning from cardiopulmonary bypass supported by norepinephrine and volume substitution is commenced. The ventilator is adjusted to pressure-controlled ventilation with a positive end-expiratory pressure (PEEP) of $10 \mathrm{cmH}_{2} \mathrm{O}$ and a minute volume allowing some degree of hyperventilation. The advantage of pressure-controlled ventilation is the early detection of a decreasing tidal volume in the case of endobronchial haemorrhage. In case of high pulmonary vascular resistance (PVR) preoperatively or limited success of endarterectomy, inhaled Iloprost (a prostaglandin derivate) is administered. Routine decannulation and sternal closure ensues, and the operation is completed in the usual manner. The patient is ventilated until the next morning and after gradually reducing the PEEP, extubation ensues.

\section{Alternatives}

Around two thirds of CTEPH-patients are amenable for surgery even if the obstructions are more distally located. For the inoperable patients, medical treatments with PAH-pharmacotherapy and/or BPA are recommended treatment options (7). Therefore, treatment of CTEPH patients belongs in specialized centers with experience in all 


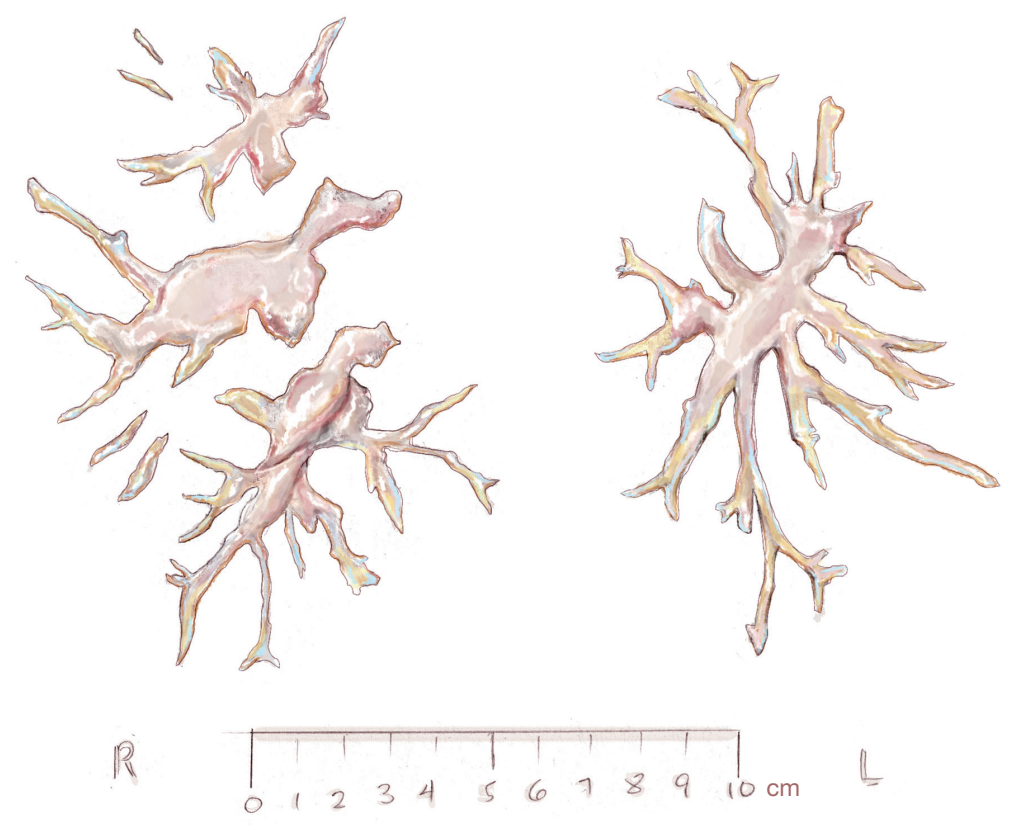

Figure 11 Endarterectomy specimen of the right and left pulmonary arteries with a ruler (1 to $10 \mathrm{~cm})$.

therapeutic modalities. Figure 12 depicts a schematic view of the pulmonary vascular tree and the most appropriate levels of the different treatment.

\section{Comments}

\section{Clinical results}

Our experience with this operation began in 1989 and since then we have undertaken approximately 2,400 PEA procedures. Since 2009, the vast majority of patients were operated on in the Kerckhoff-Clinic in Bad Nauheim $(n=1,438)$. Before the corona epidemic, the annual number of operations amounted to approximately 150 to 160 . The mortality dropped for the last 5 years to $2.1 \%(\mathrm{n}=712)$. Complication rates are on average $60.1 \%$, most complications involve cardiac arrhythmias and mild reperfusion edema. Severe complications like pronounced reperfusion edema, cerebral bleeding or stroke, and endobronchial bleeding occurred in $11.2 \%$ of the patients. In a cohort representing the year 2014 and 2015, 248 operative patients were evaluated before and after PEA (8). The mean pulmonary arterial pressure (MPAP) and the PVR decreased from 43 [interquartile range (IQR), 34-59] to 29 (IQR, 26-33) $\mathrm{mmHg}(\mathrm{P}<0.001)$ and from 7.2 (IQR, 5.0-10.3) to 4.8 (IQR, 3.5-6.4) WU $(\mathrm{P}<0.001)$, respectively.
With the start of our BPA-program in 2014, in highly selected cases, we combined PEA with BPA as a hybrid procedure if patients had severe hemodynamic impairment and a challenging surgical disease pattern. In these cases, one side (usually right) seemed amenable for surgery whereas the remaining side had a more distal distribution of the obstructions. We reported our first three patients with this combined approach in 2016 and planned for a further 20 patients (9).

Cannon et al. reported that after PEA, a notable number (up to $50 \%$ ) of patients will exhibit recurrent or persistent $\mathrm{PH}$ (10). They highlighted that a MPAP of $\geq 38 \mathrm{mmHg}$ and a PVR $\geq 5.7 \mathrm{WU}\left(425 \mathrm{dyn} \cdot \mathrm{s} \cdot \mathrm{cm}^{-5}\right.$ ) correlates with poorer long-term survival rates. Therefore, it is mandatory that patients are closely monitored by $\mathrm{PH}$ centers and multimodal therapy considered to improve symptoms and perhaps to improve the survival rates of CTEPH patients.

In patients with pulmonary arterial obstruction with almost normal hemodynamic parameters at rest, but shortness of breath on exertion, chronic thromboembolic pulmonary disease (CTEPD) without PH is diagnosed (7). In highly selected cases, we offer these patients a treatment by PEA with promising results (11-13). We were able to demonstrate that total pulmonary resistance and the slope of the MPAP-cardiac output-relation decreases significantly after PEA and this was accompanied by an improved quality of life (11). 


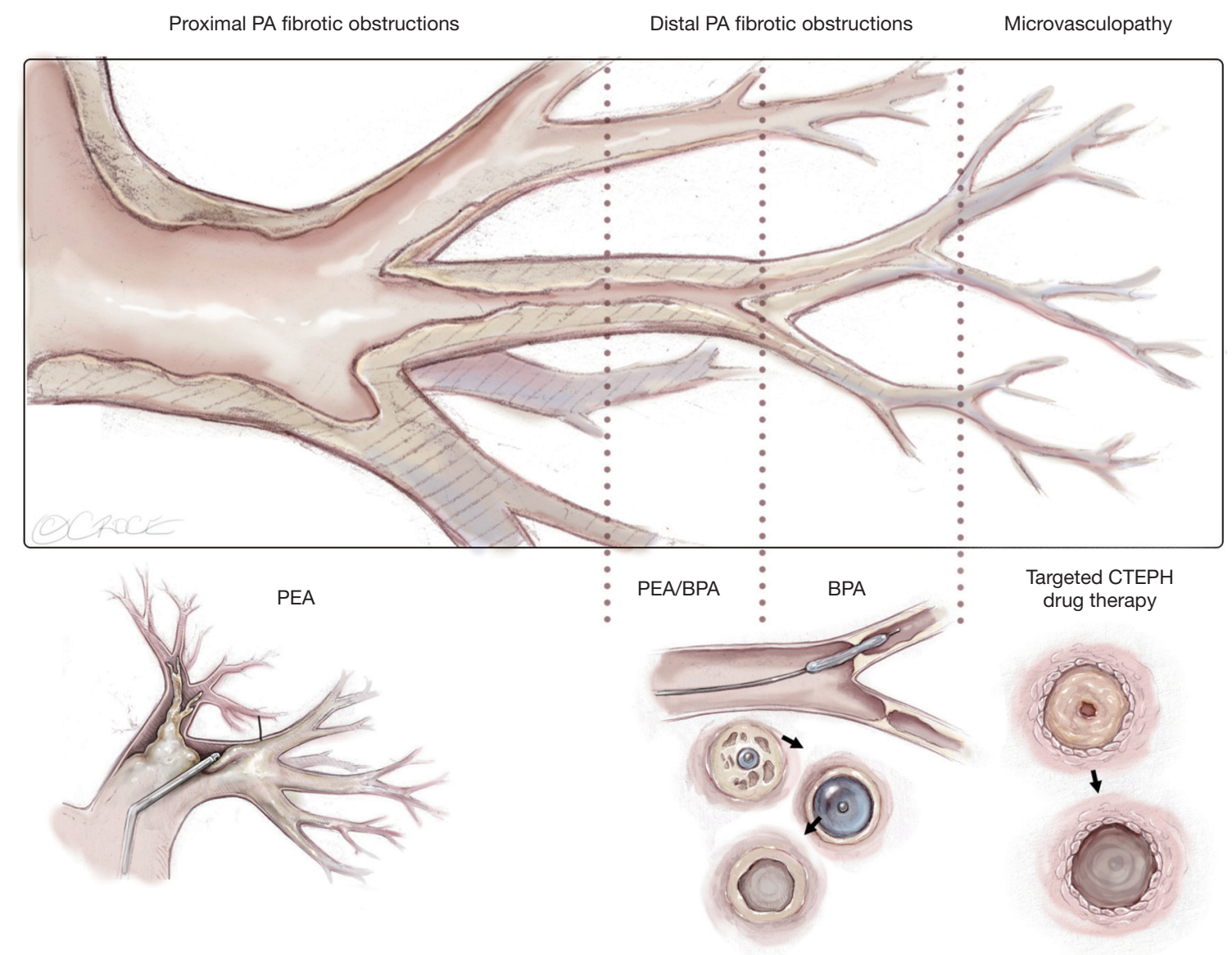

Figure 12 Schematic depiction of the therapeutic modalities for CTEPH-patients: proximal arteries are the domain for pulmonary endarterectomy, far distally medical pulmonary artery hypertension medication is the treatment of choice and in between BPA has its place. CTEPH, chronic thromboembolic pulmonary hypertension; BPA, balloon pulmonary angioplasty; PEA, pulmonary endarterectomy; PA, pulmonary artery.

In conclusion, PEA remains the recommended treatment for patients suffering from CTEPH, which is underlined by a recent report of the second worldwide CTEPH registry (1). This demanding surgery should be done in specialized CTEPH centers with experienced PEA surgeons. Historically, it has been considered that an expert center should perform a minimum of 20 PEAs per year with a mortality rate less than $10 \%$. Meanwhile more treatment options are available and therefore the definition of expert centers becomes more important. Data from the first international CTEPH registry showed that mortality was related to the caseload of a center, where centers with at least 50 PEAs per year reported a mortality of 3.4\% (14). Another retrospective single-center series with consecutive cases reported a significant reduction in mortality between an earlier (1,000 cases, 5.2\%) and a later (500 cases, $2.2 \%)$ timeframe (15). Meanwhile, a consensus was reached that an expert center should perform at least 50 PEAs per year for a population of 40-50 million adults with $\geq 5$ years' experience and mortality rates should be consistently below $5 \%$. Additionally, surgeons should be able to perform segmental and subsegmental endarterectomy and the center should also offer BPA and medical therapy for inoperable patients (16).

In conclusion, we can state that PEA has a steep and long learning curve and surgeons need at least 50 mentored PEA procedures in a high-volume center with selected cases before they are able to perform PEA in their own right (16).

\section{Pitfalls and caveats}

There are several pitfalls in PEA surgery and some of them 
may have life-threatening consequences. During evaluation, it is important to recognize patients with (idiopathic) PAH with in-situ thrombosis, particularly of the central pulmonary arteries. In such cases, the removal of centrally located material does not change PVR and weaning from ECC is rarely possible, equating to high risk of mortality. A similar situation arises when PEA in a patient with severe $\mathrm{PH}$ is incomplete and the distally located obstructions are left in situ.

Another important technical pitfall is the incision line of the right pulmonary artery. If it is localized too far cranially, it might be extended into the first branch of the upper lobe. Endarterectomy may cause further tearing in this locale, making the closure of the pulmonary artery quite difficult. Especially if the suture line is under tension and the vessel wall is fragile, closure may only be possible using a pericardial patch.

If severe endobronchial hemorrhage occurs during weaning from the ECC, weaning is not possible. One option is endobronchial blocking of the affected bronchus. Another option is to switch to a short-term veno-arterial heparin-coated ECMO system, allowing restoration of the coagulation system. In our experience, this is a very successful maneuver that leads to sealing of the tiny vascular-bronchial connections and enables weaning from the ECMO system after a short period (17).

\section{Acknowledgments}

The authors wish to acknowledge and thank Beth Croce for preparing the final illustrations for this publication.

Funding: None.

\section{Footnote}

Conflicts of Interest: The authors have no conflicts of interest to declare.

Open Access Statement: This is an Open Access article distributed in accordance with the Creative Commons Attribution-NonCommercial-NoDerivs 4.0 International License (CC BY-NC-ND 4.0), which permits the noncommercial replication and distribution of the article with the strict proviso that no changes or edits are made and the original work is properly cited (including links to both the formal publication through the relevant DOI and the license). See: https://creativecommons.org/licenses/by-nc-nd/4.0/.

\section{References}

1 Guth S, D'Armini AM, Delcroix M, et al. Current strategies for managing chronic thromboembolic pulmonary hypertension: results of the worldwide prospective CTEPH Registry. ERJ Open Res 2021;7:e00850-2020.

2. Galiè N, Humbert M, Vachiery JL, et al. 2015 ESC/ERS Guidelines for the diagnosis and treatment of pulmonary hypertension: The Joint Task Force for the Diagnosis and Treatment of Pulmonary Hypertension of the European Society of Cardiology (ESC) and the European Respiratory Society (ERS): Endorsed by: Association for European Paediatric and Congenital Cardiology (AEPC), International Society for Heart and Lung Transplantation (ISHLT). Eur Heart J 2016;37:67-119.

3. Delcroix M, Lang I, Pepke-Zaba J, et al. Long-Term Outcome of Patients With Chronic Thromboembolic Pulmonary Hypertension: Results From an International Prospective Registry. Circulation 2016;133:859-71.

4. Madani MM. Pulmonary endarterectomy for chronic thromboembolic pulmonary hypertension: state-of-the-art 2020. Pulm Circ 2021;11:20458940211007372.

5. Wiedenroth CB, Liebetrau C, Gall $\mathrm{H}$, et al. The effective systematic heparin pre-treatment on thrombus formation on pulmonary artery catheter tips during pulmonary endarterectomy for chronic thromboembolic pulmonary hypertension: a randomized, double-blind study. J Thromb Thrombolysis 2017;44:335-40.

6. Morsolini M, Azzaretti A, Orlandoni G, et al. Airway bleeding during pulmonary endarterectomy: the "bubbles" technique. J Thorac Cardiovasc Surg 2013;145:1409-10.

7. Delcroix M, Torbicki A, Gopalan D, et al. ERS statement on chronic thromboembolic pulmonary hypertension. Eur Respir J 2021;57:2002828.

8. Lankeit M, Krieg V, Hobohm L, et al. Pulmonary endarterectomy in chronic thromboembolic pulmonary hypertension. J Heart Lung Transplant 2018;37:250-8.

9. Wiedenroth CB, Liebetrau C, Breithecker A, et al. Combined pulmonary endarterectomy and balloon pulmonary angioplasty in patients with chronic thromboembolic pulmonary hypertension. J Heart Lung Transplant 2016;35:591-6.

10. Cannon JE, Su L, Kiely DG, et al. Dynamic Risk Stratification of Patient Long-Term Outcome After Pulmonary Endarterectomy: Results From the United Kingdom National Cohort. Circulation 2016;133:1761-71. 
11. Guth S, Wiedenroth CB, Rieth A, et al. Exercise right heart catheterisation before and after pulmonary endarterectomy in patients with chronic thromboembolic disease. Eur Respir J 2018;52:1800458.

12. van Kan C, van der Plas MN, Reesink HJ, et al. Hemodynamic and ventilatory responses during exercise in chronic thromboembolic disease. J Thorac Cardiovasc Surg 2016;152:763-71.

13. Taboada D, Pepke-Zaba J, Jenkins DP, et al. Outcome of pulmonary endarterectomy in symptomatic chronic thromboembolic disease. Eur Respir J 2014;44:1635-45.

14. Mayer E. Surgical and post-operative treatment of chronic thromboembolic pulmonary hypertension. Eur Respir Rev
2010;19:64-7.

15. Madani MM, Auger WR, Pretorius V, et al. Pulmonary endarterectomy: recent changes in a single institution's experience of more than 2,700 patients. Ann Thorac Surg 2012;94:97-103; discussion 103.

16. Jenkins D, Madani M, Fadel E, et al. Pulmonary endarterectomy in the management of chronic thromboembolic pulmonary hypertension. Eur Respir Rev 2017;26:160111.

17. Guth S, Wiedenroth CB, Wollenschläger M, et al. Shortterm venoarterial extracorporeal membrane oxygenation for massive endobronchial hemorrhage after pulmonary endarterectomy. J Thorac Cardiovasc Surg 2018;155:643-9.

Cite this article as: Guth S, Mayer E, Prüfer D, Wiedenroth CB. Pulmonary endarterectomy: technique and pitfalls. Ann Cardiothorac Surg 2022;11(2):180-188. doi: 10.21037/acs-2021pte-185 